\title{
DECOLORACIÓN DE ALGINATO DE SODIO EXTRAÍDO DE LAS ALGAS PARDAS MARINAS DEL GÉNERO Sargassum CON EL USO DE PERÓXIDO DE HIDRÓGENO
}

\section{Erik L. Regalado y Abilio Laguna}

Centro de Bioactivos Marinos, Ministerio de Ciencia Tecnología y Medio Ambiente, Loma y 37, Alturas del Vedado, Ciudad Habana, Cuba

\section{Mabel Torres*}

Farmácia de Manipulação Valfarma, Av. Carlos Gomes, 1987, 78901-350 Porto Velho - RO, Brasil

\section{Juan D. Sabatier}

Facultad de Química e Ingeniería Química, Universidad Nacional Mayor de San Marcos, Ciudad Universitaria,

Av. Venezuela s/n, Lima 1, Peru

\section{Margarita Hernández y Anselmo E. Ferrer}

Centro de Biomateriales, Universidad de La Habana, Plaza de la Revolución, Ciudad de La Habana, Cuba

\section{Clara Nogueiras}

Centro de Productos Naturales, Zapata y Carlos III, Universidad de La Habana, Plaza de la Revolución,

Ciudad de La Habana, Cuba

Recebido em 6/5/05; aceito em 7/4/06; publicado na web em 22/8/06

\begin{abstract}
BLEACHING OF SODIUM ALGINATE FROM THE BROWN SEAWEEDS SARGASSUM WITH HYDROGEN PEROXIDE. Hydrogen peroxide bleaching of sodium alginate from seaweeds oh the Sargassum genus was studied. The influence of $\mathrm{H}_{2} \mathrm{O}_{2}$ concentration (percentage of $\mathrm{H}_{2} \mathrm{O}_{2}$ on a dry weight alginate basis, w/w) and $\mathrm{NaOH} / \mathrm{H}_{2} \mathrm{O}_{2}$ ratio $\left(\% \mathrm{NaOH} / \% \mathrm{H}_{2} \mathrm{O}_{2}\right.$, both referred to a dry weight alginate basis, w/w) on the molecular weight, color removal and content of $\mathrm{Fe}^{3+}$ ions of bleached alginate samples was investigated by UV and IR spectroscopies, colorimetric determination of $\mathrm{Fe}^{3+}$ ions and vapor pressure osmometry. Higher yield, purity and molecular weight of alginate were obtained using $3 \%$ (or less) of hydrogen peroxide and a $\mathrm{NaOH} / \mathrm{H}_{2} \mathrm{O}_{2}$ ratio of 1.2 for bleaching.
\end{abstract}

Keywords: bleaching; sodium alginate; Sargassum.

\section{INTRODUCCIÓN}

En la actualidad tanto el ácido algínico como sus sales son empleados en diversas ramas de la industria y la ciencia, tal es el caso de la industria alimenticia, la medicina y la industria farmacéutica entre otras ${ }^{1}$. En odontología se utiliza como uno de los componentes en los materiales de impresión dental, para cubrir y proteger dentaduras de resina acrílica ${ }^{2}$; además se reporta el uso de alginatos para encapsular diversos principios activos ${ }^{3}$, entre estos se encuentran fármacos como la nicardipina, el metronidazol y la teofilina ${ }^{4}$.

Sin embargo, la coloración que presenta el alginato después de ser extraído limita sus posibilidades de uso y se hace necesario someterlo a un proceso de decoloración o blanqueo. El blanqueo de materiales celulósicos data de la antigüedad y se realizaba hirviéndolos en una solución alcalina de la cual eran posteriormente sacados y se sometían a la acción del sol para finalmente ser lavados con abundante agua ${ }^{5}$.Esto fue así hasta que Scheele descubrió el cloro elemental en el año 1774, demostrando que el cloro tenía una poderosa acción blanqueante sobre las fibras vegetales ${ }^{6}$.

Recientemente Ochoa y colaboradores ${ }^{7}$ realizaron un estudio de decoloración de alginato de sodio usando como agente decolorante hipoclorito de sodio, con el cual obtuvieron buenos resultados en cuanto a color, sin embargo, la utilización del cloro y sus derivados presenta serias desventajas entre las que podemos mencionar: el encarecimiento del equipamiento tecnológico; por tener que estar construido de materiales resistentes a la corrosión, la contaminación ambiental que se origina producto de los compuestos tóxicos y bioacumulativos que

*e-mail: esther1920@yahoo.es se generan y el marcado efecto de degradación que han mostrado sobre el alginato. Esto ha motivado la búsqueda y utilización de alternativas no contaminantes como son: el ozono, el carbón activado y los peróxidos que provocan menor efecto degradativo sobre los polisacáridos en general y destruyen las sustancias coloreadas, garantizando una excelente estabilidad de blancura ${ }^{6,8}$.

El peróxido de hidrógeno es un fuerte agente oxidante y decolorante, además no presenta los problemas de liberación de gases o residuos químicos contaminantes que están asociados con otros oxidantes ${ }^{8}$. Tradicionalmente, la decoloración con peróxidos se realiza durante el proceso de extracción del material, lo cual dificulta el ajuste de las condiciones necesarias para su uso, entre estas se encuentra el pH del medio de reacción; el cual no debe ser mayor que 11,5 para que no se favorezca la conjugación de especies cromofóricas. Por otra parte, se hace imposible conocer la concentración a la que se encuentra el material a decolorar, para así ajustar las cantidades de $\mathrm{H}_{2} \mathrm{O}_{2}$ a usar. Finalmente, no se pueden controlar los iones metálicos presentes en el medio de reacción, los cuales traen como consecuencia la descomposición del $\mathrm{H}_{2} \mathrm{O}_{2}$ y la degradación del material.

En Cuba, la materia prima para obtener ácido algínico y sus sales son algas en su mayoría del género Sargassum (Phaeophyta, Fucales), que provenientes del Mar de los Sargazos arriban fundamentalmente a las siguientes regiones del país: Cayo Coco, Cayo Largo del Sur, Caibarién, Santiago de Cuba, Cabo Cruz y Playa Santa Lucia ${ }^{9}$. En el presente trabajo se estudia la decoloración con $\mathrm{H}_{2} \mathrm{O}_{2}$ de alginato de sodio sin previo tratamiento de blanqueo durante el proceso de extracción, con vistas a obtener alginatos de menos coloración y mayor viscosidad. 


\section{PARTE EXPERIMENTAL}

\section{Equipos utilizados}

El $\mathrm{pH}$ del sistema reaccionante se determinó en un pH-metro digital HI 8521 Hanna Instruments. Los espectros UV se realizaron en un espectrofotómetro Pharmacia Ultrospec III y los espectros IR en un espectrofotómetro Ati Mason Genesis FTIR, de rango espectral 4000 a $400 \mathrm{~cm}^{-1}$. Las determinaciones de $\mathrm{Fe}^{3+}$ se realizaron en un espectrofotómetro GBC UV-Visible Cintra 10e y los pesos moleculares se determinaron en un osmómetro de fase vapor Knauer de alta sensibilidad.

\section{Diseño experimental}

Se planificó un bloque experimental factorial $2^{2}$ con un centro para un total de 5 experimentos, en los que se variaron la concentración de peróxido (porcentaje referido al peso de alginato en base seca, $\%$ p/p) y la relación $\mathrm{NaOH} / \mathrm{H}_{2} \mathrm{O}_{2}$ (relación entre los porcentajes de $\mathrm{NaOH}$ y $\mathrm{H}_{2} \mathrm{O}_{2}$ en el sistema, referidos ambos respecto al peso de alginato en base seca, \% p/p) según se muestra en las Tablas 1 y 2.

Tabla 1. Variables independientes y niveles de estudio

\begin{tabular}{llccc}
\hline Variables & Símbolo & -1 & 0 & +1 \\
\hline Concentración de $\mathrm{H}_{2} \mathrm{O}_{2} *$ & $\mathrm{H}_{2} \mathrm{O}_{2}\left(\mathrm{X}_{1}\right)$ & 2 & 3 & 4 \\
Relación \% $\mathrm{NaOH} / \% \mathrm{H}_{2} \mathrm{O}_{2} * *$ & $\mathrm{NaOH} / \mathrm{H}_{2} \mathrm{O}_{2}\left(\mathrm{X}_{2}\right)$ & 1 & 1,2 & 1,4 \\
\hline
\end{tabular}

* \% p/p respecto al alginato en base seca; ** ambos porcentajes $\mathrm{p} / \mathrm{p}$ referidos al alginato base seca

Tabla 2. Matriz experimental del diseño

\begin{tabular}{lcccc}
\hline Exp. & $\mathrm{X}_{1}$ & $\mathrm{X}_{2}$ & $\mathrm{H}_{2} \mathrm{O}_{2}, \%$ & $\mathrm{NaOH} / \mathrm{H}_{2} \mathrm{O}_{2}$ \\
\hline $\mathbf{1}$ & -1 & -1 & 2 & 1 \\
$\mathbf{2}$ & 1 & -1 & 4 & 1 \\
$\mathbf{3}$ & -1 & 1 & 2 & 1,4 \\
$\mathbf{4}$ & 1 & 1 & 4 & 1,4 \\
$\mathbf{5}$ & 0 & 0 & 3 & 1,2 \\
\hline
\end{tabular}

\section{Decoloración del alginato de sodio}

La decoloración se realizó aplicando la correspondiente concentración de $\mathrm{H}_{2} \mathrm{O}_{2}$ sobre una dispersión de alginato de sodio al $2 \%$ de consistencia ( 2 gramos de alginato de sodio en $100 \mathrm{~mL}$ de dispersión) manteniendo el sistema a la temperatura de $80{ }^{\circ} \mathrm{C}$ y con agitación constante. Previo a la adición del peróxido fueron añadidos a la dispersión de alginato las cantidades adecuadas de silicato de sodio y sulfato de magnesio para lograr concentración del 4 y 0,05\%, respectivamente, de estos reactivos referidos al peso de alginato en base seca. Esto se realizó para garantizar una acción secuestrante sobre los iones metálicos posiblemente presentes con vistas a minimizar su acción catalítica en la degradación oxidativa del alginato en medio alcalino.

Posteriormente se adicionó la cantidad apropiada de $\mathrm{NaOH}$ según el diseño experimental y finalmente se procedió a añadir el $\mathrm{H}_{2} \mathrm{O}_{2}$ (Tabla 3). Este momento fue considerado el tiempo cero de la reacción comenzándose a valorar el peróxido de hidrógeno residual indirectamente con tiosulfato de sodio $0,01 \mathrm{~N}$ cada 5 min hasta que el porcentaje de peróxido residual estuviera entre un 10 y un $15 \%$ con respeto al inicial. Alcanzado este punto se anotó el tiempo de reacción y se adicionó una solución de ácido clorhídrico $4 \mathrm{~N}$ hasta $\mathrm{pH}$ entre 2 y 3 para precipitar el ácido algínico el cual se suspendió en etanol y se convirtió en alginato de sodio por adición de una disolución saturada de carbonato de sodio hasta valores de $\mathrm{pH}$ entre 7 y 8 . El alginato de sodio obtenido fue filtrado a gravedad a través de una malla y posteriormente colocado en una estufa a vacío a $60{ }^{\circ} \mathrm{C}$ por $24 \mathrm{~h}$. En todos los experimentos se calcularon los rendimientos.

Tabla 3. Volúmenes de $\mathrm{NaOH}$ y $\mathrm{H}_{2} \mathrm{O}_{2}$ añadidos por experimento

\begin{tabular}{lcc}
\hline Exp. & $\mathrm{V}_{\mathrm{NaOH}}(\mathrm{mL})$ & $\mathrm{V} \mathrm{H}_{2} \mathrm{O}_{2}(\mathrm{~mL})$ \\
\hline $\mathbf{1}$ & 0,27 & 0,14 \\
$\mathbf{2}$ & 0,53 & 0,27 \\
$\mathbf{3}$ & 0,37 & 0,14 \\
$\mathbf{4}$ & 0,75 & 0,27 \\
$\mathbf{5}$ & 0,48 & 0,20 \\
\hline
\end{tabular}

\section{Remoción de color}

Para la evaluación de la remoción de color se registraron espectros UV en un rango espectral de 200 a $800 \mathrm{~nm}$ utilizando cubetas de $1 \mathrm{~cm}$ de paso óptico. Se prepararon para el estudio disoluciones acuosas al $0,1 \%(\mathrm{p} / \mathrm{v})$ de alginato de sodio comercial (Macrocystis pyrifera, Sigma), del alginato de sodio de partida y de las muestras decoloradas.

\section{Espectroscopia infrarroja}

Los espectros IR de los alginatos decolorados se determinaron en pastillas de $\mathrm{KBr}$ a una concentración aproximada de $2 \mathrm{mg}$ de muestra/100 mg KBr.

\section{Determinación del porcentaje de hierro}

La determinación del $\mathrm{Fe}^{3+}$ se realizó mediante colorimetría, utilizando como reactivo desarrollador de color la ortofenantrolina. Se obtuvo una curva de calibración usando como patrón $\mathrm{FeCl}_{3}$, en un rango de concentraciones que varió de 1 a 8,446 ppm. Se realizaron 3 réplicas para cada muestra.

\section{Determinación de pesos moleculares por osmometría en fase vapor}

La calibración del equipo se realizó empleando disoluciones de lactosa monohidratada como patrón, en una cámara saturada con vapor de agua. Se prepararon soluciones a diferentes molalidades $(1,45$; 1,$62 ; 1,86 ; 2,01 ; 2,23 \mathrm{~g} / \mathrm{kg}$ ) del patrón y de cada unos de los alginatos de sodio obtenidos en el diseño experimental. Se determinó experimentalmente la diferencia de temperatura entre los dos termistores, los cuales corresponden al solvente y a la muestra a diferentes molalidades. A través de un gráfico DT/molalidad vs molalidad se extrapoló el intercepto y se determinó el peso molecular del alginato de sodio para cada experimento por equivalencia teniendo en cuenta el intercepto del patrón cuya masa molecular es conocida.

\section{RESULTADOS Y DISCUSIÓN}

\section{Decoloración del alginato de sodio}

A continuación, en la Tabla 4, se muestran los resultados de las condiciones experimentales que estuvieron bajo control en cada ensayo y los rendimientos alcanzados.

Como se puede apreciar en todos los experimentos del diseño hay una ligera disminución del $\mathrm{pH}$ del sistema, este comportamiento está dentro de lo esperado ya que a pesar de la formación de grupos carboxílicos durante el proceso de decoloración que debieran pro- 
Tabla 4. Resultados de las condiciones experimentales bajo control durante el proceso de decoloración y rendimientos obtenidos

\begin{tabular}{lccccc}
\hline Resultados & Exp 1 & Exp 2 & Exp 3 & Exp 4 & Exp 5 \\
\hline pH inicial & 10,7 & 11,4 & 11,2 & 11,5 & 11,3 \\
pH final & 10,3 & 11,1 & 10,6 & 11,2 & 11,2 \\
Tiempo decoloración (min.) & 55 & 60 & 40 & 70 & 55 \\
$\mathrm{H}_{2} \mathrm{O}_{2 \text { Residual }}(\%)$ & 12,0 & 11,4 & 12,4 & 14,1 & 13,9 \\
Rendimiento (\%) & 63,7 & 45,0 & 73,2 & 47,7 & 64,2 \\
\hline
\end{tabular}

vocar una disminución del pH, el sistema se encuentra en presencia de un buffer (silicato de sodio/ $\mathrm{NaOH}$ ) que mantiene el $\mathrm{pH}$ en valores óptimos para la decoloración.

Los mejores rendimientos, por encima del $60 \%$, se aprecian cuando se emplean las menores concentraciones de $\mathrm{H}_{2} \mathrm{O}_{2}$ correspondientes a 2 y $3 \%$. Con los valores codificados que se muestran en la matriz del diseño experimental y los rendimientos obtenidos para cada experimento, se realizó un análisis de regresión múltiple y se obtuvo la siguiente Ecuación:

Rendimiento $=59,27-11,69 * X_{1}$

A pesar de que la $\mathrm{F}_{\text {Fischer }}$ experimental $(28,18)$ es ligeramente menor que la $\mathrm{F}_{\text {Fischer }}$ tabulada $(34,1)$, se puede decir que el modelo ajusta ya que el coeficiente de correlación experimental $\left(\mathrm{R}_{\text {exp. }}=\right.$ 90,38 ) es mayor que el tabulado $\left(r_{\text {crítico }}=87,8\right)$, para 3 grados de libertad, y $\mathrm{n}=5$. Con lo que se confirma que el rendimiento depende de la concentración de peróxido de hidrógeno usada en el proceso de decoloración, cuestión que había sido observada a priori y que se explica por la acción degradante que también tiene este agente decolorante. Ochoa et al. ${ }^{7}$ obtuvo resultados semejantes al realizar la decoloración de alginato de sodio con hipoclorito de sodio pues al añadirse una mayor concentración de agente decolorante (superior a $15 \%$ ) se obtuvo como resultado final una degradación apreciable con bajos rendimientos.

\section{Remoción de color}

El análisis de los espectros de absorción del alginato de partida y la muestra comercial permitió determinar que la sección entre 250 y 320 $\mathrm{nm}$ es la de mayor interés para este trabajo, debido a que en esta zona es donde único se aprecia diferencia entre ambos, en el alginato de partida sin decolorar aparece una amplia banda de absorción en esta sección que no se observa en el alginato comercial. En la Figura 1 mostramos la sección seleccionada de los espectros de absorción de las muestras decoloradas y del alginato de sodio de partida.

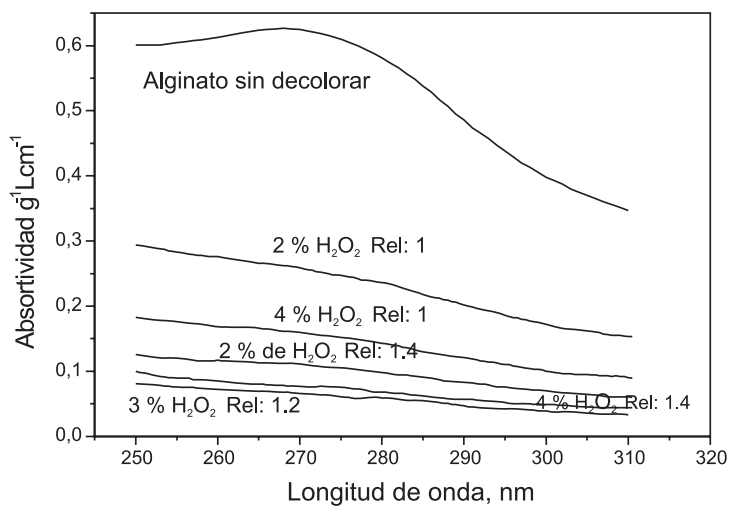

Figura 1. Espectro de absorción (250-320 nm) del alginato de sodio de partida y las muestras decoloradas
Como se puede apreciar la absorción de las muestras en el UV disminuye en la medida que se usan mayores concentraciones del agente decolorante. Los mejores resultados en cuanto a coloración también se obtienen con las más altas concentraciones $\mathrm{de}_{2} \mathrm{O}_{2}$, aunque es necesario señalar que no se aprecia una marcada diferencia entre los resultados obtenidos con las concentraciones de 3 y $4 \%$, por lo cual puede considerarse que no es necesario usar soluciones de peróxido con concentraciones superiores al 3\%. Los mejores resultados se obtienen cuando se trabaja bajo las condiciones del experimento central del diseño, o sea, $\mathrm{H}_{2} \mathrm{O}_{2}$ al $3 \%$ y relación $\mathrm{NaOH} / \mathrm{H}_{2} \mathrm{O}_{2}$ de 1,2 .

\section{Espectroscopia infrarroja}

El mecanismo de degradación de los polisacáridos por la acción de un agente oxidante en medio alcalino se ve marcado al inicio por un incremento del contenido de grupos carbonilos, los cuales pasan posteriormente a carboxilatos y por último se produce la fragmentación de las cadenas que se evidencia por la disminución de viscosidad y, en caso extremo, por una afectación del rendimiento ${ }^{10}$. Para el cálculo de la intensidad relativa de la banda del grupo carboxilato se tomó como referencia la banda de 1026-1032 $\mathrm{cm}^{-1}$ la cual corresponde al grupo $v_{\mathrm{C}-\mathrm{O}} \mathrm{y} \mathrm{v}_{\mathrm{C}-\mathrm{C}}$ del anillo carbohidrático.

En la Figura 2 se puede apreciar la variación de la intensidad relativa de la banda del grupo carboxilato al variar la cantidad del agente decolorante, para una relación $\mathrm{NaOH} / \mathrm{H}_{2} \mathrm{O}_{2}$ de 1 , o sea, al aumentar la cantidad de agente oxidante aumenta el número de grupos carboxilato y por lo tanto aumenta su intensidad relativa, lo cual corrobora la primera parte del mecanismo de degradación oxidativa propuesto para los carbohidratos ${ }^{10}$. Comportamiento semejante fue observado por Ochoa et $a .^{7}$ durante a decoloración de alginato con hipoclorito de sodio.

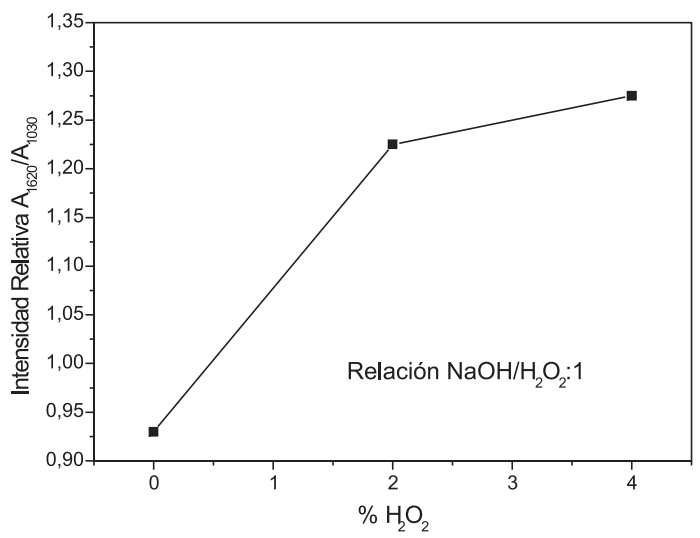

Figura 2. Influencia de la dosis de peróxido sobre la intensidad relativa de la banda del grupo carboxilato

\section{Determinación del porcentaje de hierro $\mathrm{Fe}^{3+}$}

La presencia de cationes metálicos como el $\mathrm{Fe}^{3+}$ tienen gran importancia dentro del proceso de decoloración, ya que estos tienden a precipitar como óxidos o hidróxidos coloidales cuando se trabaja en medio alcalino y actúan como catalizadores de la descomposición del peróxido de hidrógeno, así como la degradación y posterior reversión de color del material ${ }^{11}$.

Es por ello que en el proceso de decoloración con peróxido de hidrógeno, se emplean agentes quelatantes tales como: EDTA, DTPA, DTMPA o silicato de magnesio a fin de disminuir su concentración ${ }^{8}$. En la Tabla 5 se muestran los valores obtenidos en las determinaciones de $\mathrm{Fe}^{3+}$ para el alginato sin decolorar y el alginato decolorado siguiendo las condiciones del experimento 5 . 
Tabla 5. Concentraciones de $\mathrm{Fe}^{3+}$ determinadas en el alginato de partida y en una de las muestras decoloradas

\begin{tabular}{lcc}
\hline Muestras & Concentración (ppm) & $\mathrm{Fe}^{3+}(\%)$ \\
\hline Alginato de Partida & 3,524 & 4,4 \\
Alginato decolorado(Exp 5) & 1,834 & 2,3 \\
\hline
\end{tabular}

Al comparar la concentración de $\mathrm{Fe}^{3+}$ en el alginato de partida con la del alginato decolorado puede observarse que el valor del primero casi duplica al segundo. Los resultados concuerdan con lo esperado, debido a que en el proceso de decoloración se forma in situ el silicato de magnesio que acompleja parte de los iones metálicos presentes, reduciéndose de esta manera la concentración de $\mathrm{Fe}^{3+}$ en el alginato de sodio decolorado finalmente obtenido.

\section{Determinación de pesos moleculares por osmometría en fase vapor}

El conocimiento del peso molecular es de gran interés para determinar el futuro uso práctico de los polímeros en general, por ello resulta importante determinar los pesos moleculares de los alginatos obtenidos en cada experimento y de esta forma conocer la influencia de las condiciones experimentales sobre los mismos. Los resultados de la determinación de los pesos moleculares se muestran en la Figura 3 y en la Tabla 6.

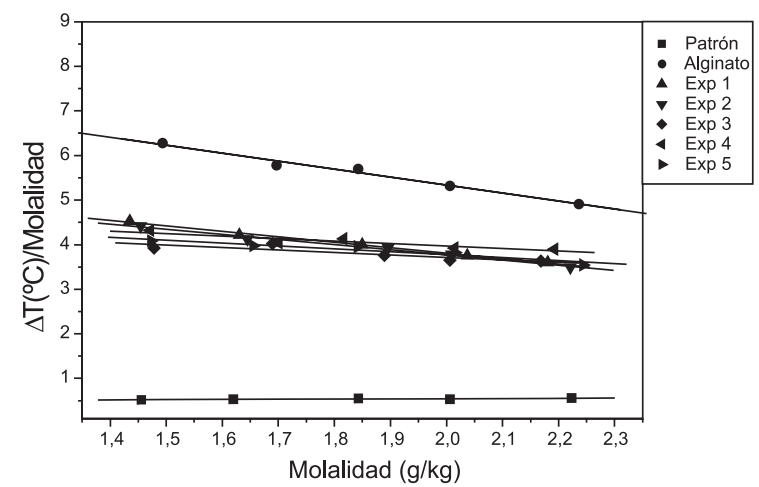

Figura 3. Gráfico de determinación de las masas moleculares promedio en número (Mn) mediante el método de osmometría en fase vapor

Tabla 6. Masas moleculares promedio en número (Mn) determinadas

\begin{tabular}{lccc}
\hline Muestras & $\mathrm{H}_{2} \mathrm{O}_{2}(\%)$ & Intercepto & $\mathrm{Mn}$ (Dalton) \\
\hline Patrón & - & 0,4693 & 36,032 \\
Alginato inicial & - & 8,9162 & 6,846 \\
Exp I & 2 & 6,2571 & 4,805 \\
Exp II & 4 & 6,0692 & 4,660 \\
Exp III & 2 & 4,8165 & 3,698 \\
Exp IV & 4 & 5,0829 & 3,903 \\
Exp V & 3 & 5,5027 & 4,225 \\
\hline
\end{tabular}

Como se puede apreciar los valores de masa moleculares están por debajo de lo reportado en la literatura para el género Sargassum (Mn 36.032 Dalton) $)^{12}$, lo cual pudo estar influenciado por la presencia de iones metálicos en el medio de reacción, además el alginato de sodio de partida no era de reciente extracción y tenía un peso molecular de por sí bajo. El análisis de los resultados nos muestra que tiene mayor influencia la relación $\mathrm{NaOH} / \mathrm{H}_{2} \mathrm{O}_{2}$ que la concentración de peróxido. Al comparar el valor del peso molecular de la muestra del experimento 1 con el de la 2 (tienen igual $\mathrm{NaOH} /$ $\mathrm{H}_{2} \mathrm{O}_{2}$ ) vemos que hay una menor diferencia y que si lo hacemos entre este y el de la 3 (mayor $\mathrm{NaOH} / \mathrm{H}_{2} \mathrm{O}_{2}$ ) esto indica que la degradación oxidativa del polisacárido detectada a través de la disminución del peso molecular es afectada por la concentración de álcali en la mezcla reaccionante. Los resultados obtenidos en cuanto a peso molecular con el experimento central también podemos considerarlos como los mejores.

\section{CONCLUSIONES}

Mediante la técnica propuesta se logró decolorar con $\mathrm{H}_{2} \mathrm{O}_{2}$ un alginato de sodio extraído sin previa decoloración a partir de algas pardas del género Sargassum se lograron rendimientos superiores al $70 \%$. Se obtuvieron buenos resultados en cuanto a decoloración y rendimiento cuando se utiliza $\mathrm{H}_{2} \mathrm{O}_{2}$ en concentración del $3 \%$ y relación $\mathrm{NaOH} / \mathrm{H}_{2} \mathrm{O}_{2}$ de 1,2. El porcentaje de $\mathrm{Fe}^{3+}$ del alginato de sodio decolorado resultó inferior en comparación con el de partida, lo cual favorece la estabilidad del mismo. Se determinaron los pesos moleculares mediante osmometría en fase vapor, los mejores resultados se obtuvieron al usar las menores $\mathrm{NaOH} / \mathrm{H}_{2} \mathrm{O}_{2}$ y concentraciones de $\mathrm{H}_{2} \mathrm{O}_{2}$ del $3 \%$ o menores.

\section{REFERENCIAS}

1. Vidotti, E. C.; Rollemberg, M. C. E.; Quim. Nova 2004, 27, 139.

2. Metsugi, C.; Takei, N.; Arai, M.; Watanabe, T.; Jpn. Kokai Tokkyo Koho JP 2003, 11, 3058.

3. Murata, Y.; Sasaki, N.; Miyamoto, E.; Kawashima, S.; Eur. J. Pharma. Biopharm. 2000, 50, 221; Faure, E.; Leonard, M.; Laurent, A.; Dellacherie, E.; Physicochem. Engineer. Asp. 2001, 194, 221.

4. Miyazaki, S.; Kubo, W.; Attwood, D.; J. Controlled Release 2000, 67, 275.

5. Rapson, W. H.; The Bleaching of Pulp, Tappi Monograph Series, Tappi Press: New York, 1963, n. 27.

6. http://www.worldwideschool.org/Library/book/Sci/history/ AHistoryofScienceVolumeIV/Cap7.html, accesada em Março 2006.

7. Ochoa, F.; Sabatier, J.; Ferrer, A.; Int. J. Polym. Mater. 2002, 51, 745.

8. Minor, J. L.; Proceedings of Materials Research Society Symposium, San Francisco, EUA, 1992; Villar, J. C.; de la Macorra, C.; Gómez, N.; Resúmenes ampliados del $1^{\text {er }}$ Congreso Iberoamericano de Investigación en Celulosa y Papel, Puerto Iguazú, Argentina, 2000; Mao, H.; Smith, D.W.; Ozone: Sci. Eng. 1995, 17, 419.

9. Zúñiga, D.; Tesis de Maestría, Centro de Investigaciones de Ecosistemas Costeros, Cuba, 1996.

10. Ott, E.; Spurlin, H.; Grafflin, M. W.; High Polymers, Cellulose and Cellulose Derivates, Interscience Publishers: New York, 1954, vol. 5.

11. Brasileiro, L.; Colodette, J. L.; Pilo-Veloso, D.; Quim. Nova 2001, 24, 819.

12. McHugh, D. J. En Production, Properties and Uses of Alginates, Production and Utilization of Products from Commercial Seaweeds; McHugh, D. J., ed.; FAO Fisheries Technical Paper: Rome, 1987. 